\title{
DETRIMENTAL EFFECTS OF DIABETES MELLITUS ON DENTAL PULP ELEMENTS OF ALBINO RATS
}

\author{
Rania Ahmed Awwad * and Dina Hazem Gomaa **
}

\begin{abstract}
Diabetes Mellitus (DM) is a group of metabolic disorders with relative or absolute insufficiency of insulin secretion and/or concomitant resistance to the metabolic action of insulin on target tissues.

Aim: to evaluate the effect of STZ-induced-DM on the dental pulp of albino rats histologically and ultra-structurally, and its effect on pulpal T\&B lymphocytes immunohistochemically.

Methods: Thirty male albino rats, were used in this study, divided into 2 groups: Group A (Control Group): 10 rats received $2 \mathrm{ml}$ of citrate buffer (IP), was equally sub-divided into 2 subgroups A1 \& A2, according to the date of specimen collection. Group B (Experimental Group): 20 rats received an IP injection of STZ, equally sub-divided into 2 sub-groups; Sub-group B1 and B2 where the animals were sacrificed 2 and 4 weeks after establishment of DM respectively. Permanent molars of each rat were split for pulp detachment, processed for histological, immunohistochemical and ultra-structural examination.
\end{abstract}

Results: H\&E-stained sections of the pulp tissue of 2-weeks-diabetic rats showed degenerative changes. Ultra -structural examination showed degenerative changes affecting pulp elements. Immuno-histochemical examination of the pulp showed less T\&B lymphocytes.

Conclusion: DM led to degenerative changes of the pulp elements namely, cells, fibers, nerves, and vessels. DM also affected T\&B lymphocytes.

KEYWORDS: Diabetes Mellitus, Streptozotocin, Dental pulp

\section{INTRODUCTION}

Diabetes Mellitus (DM) is a group of complex multisystem metabolic disorders where there is relative or absolute insufficiency of insulin secretion and/or concomitant resistance to the metabolic action of insulin on target tissues. It was found that hyperglycaemia is the immediate metabolic consequence of DM but, ultimately, there is widespread multisystem damage. On the other hand, it is well established that the chronic hyperglycaemia of DM is associated with long-term damage, dysfunction or failure of various organs especially the eyes, kidneys, nerves, heart, and blood vessels. Most common symptoms that usually

\footnotetext{
* Ass. Prof. of Oral Biology, Faculty of Dentistry, Faculty of Dentistry, Ain Shams University ** Lecturer of Oral Biology, Faculty of Dentistry, Faculty of Dentistry, Ain Shams University
} 
appear in DM are: polydipsia, polyuria, polyphagia; excessive thirst, frequency of urination, excessive hunger (Manfredi et al, 2004).

Type I disease includes type A: immune-mediated and a type B: idiopathic DM. Type I DM is due to cell-mediated auto-immune destruction of B-cells of islets of Langerhans of the pancreas leading to complete inability of the cells to secrete insulin. It usually develops before thirty years of age, and is managed using insulin. Type II includes the most common form of DM which combines insulin resistance with an insulin secretory defect. This type is characterized by B-cells dysfunction \& inability to secrete adequate amounts of insulin, particularly after meals, and/or peripheral insulin resistance. (Atkinson and Eisenbarth, 2001; Bingley et al., 2001; Manfredi et al., 2004).

DM has been extensively studied experimentally by injecting laboratory animals with certain diabetogenic agents, such as Alloxan and Streptozotocin (STZ).

It is well established statistically that approximately $50 \%$ of patients with DM develop vascular chronic complications following years of DM. Blood vessels of all sizes are affected, from the Aorta to the smallest capillary and venule. The blood vessels are damaged by the accumulation of atheromatous deposits in the intimal tissues of the blood vessels lumen as well as altered endothelial cell permeability, leading to impaired leukotactic response (Vlassara and Palace., 2002; Manfredi et al., 2004; Catanzaro et al., 2006). The vascular changes accompanying DM lead to diabetic neuropathies; a group of nerve disorders that can be classified as peripheral, autonomic, proximal, or focal, affecting different parts of the body in various ways.

Focal neuropathy results in the sudden weakness of one nerve or a group of nerves, affecting eyes, ears, facial muscles, causing muscle weakness or pain. Wasting of connective tissues (including skin, bone, and cartilage) has historically been a prominent feature of uncontrolled DM also there is increased collagen degradation and depressed level of total collagen synthesis.

Moreover, it was found that there is great association between DM and a retarded therapeutic endodontic success at 2 years or longer postoperatively, compared to non-diabetics since it was found that there was a great association between $\mathrm{DM}$ and the predominance of obligate anaerobic micro-organisms in the root canals of diabetic patients (Iwama et al., 2006).

Many studies, comparing diabetic and nondiabetic pulps, revealed that DM caused many pulpal pathological changes, such as, angiopathies with thickened basement membranes, affecting both large and small pulp vessels. These vascular changes seemed more pronounced in the central area of the pulp. Calcifications were more frequent in diabetic pulp and were often sickle-shaped. Pulps of diabetic patients tend to age more readily because of obliterative endarteritis and because the dental pulp has limited or no collateral circulation in the fully developed tooth. Dental pulps of diabetics on occasion may manifest pain and this condition is often referred to as diabetic odontalgia (Bender et al., 2003).

Catanzaro et al., (2006) performed a study to determine the effect of DM progression on inflammatory and structural components of dental pulp in rats. They found that nitrite and kallikrein levels in dental pulp tissue were higher in diabetic rats 30 days after STZ injection than in controls, also, the concentration of collagen was decreased in diabetic rats. These results suggest that DM is a critical factor that has profound effects upon oral tissues, resulting in expression of inflammatory mediators and modifications of structural components of the dental pulp.

Accordingly, this study aimed to shed light on the detailed events and changes occurring in the dental pulp as a consequence to DM, and to evaluate the effect of STZ-induced-DM on the dental pulp 
of albino rats histologically and ultra-structurally and study the effect of Diabetes on pulpal T\&B lymphocytes immunohistochemically.

\section{MATERIALS AND METHODS}

\section{Animals}

Thirty male Albino rats, weighing around 250 grams, were used in this study. They were kept in stainless steel cages in Ain Shams animal house. Temperature and humidity conditions were controlled as possible on housing animals. They were fed standardized diet and tap water which were available ad libitum, as reviewed and approved by institution guide lines of Ain Shams University Ethical Committee. Blood sugar and body weight of all rats were recorded before the experiment. General hygiene measures were strictly applied during the whole course of the experiment.

Rats were randomly divided into 2 groups:

\section{Group A (Control Group):}

This group consisted of 10 rats that received $2 \mathrm{ml}$ of citrate buffer by intraperitoneal injections (IP). This group was equally sub-divided into 2 subgroups, according to the date of specimen collection.

Sub-group A1: The animals (5 rats) were sacrificed two weeks after establishment of DM.

Sub-group A2: The animals (5 rats) were sacrificed four weeks after establishment of DM.

\section{Group B (Experimental Group):}

This group consisted of 20 rats that received an IP injection of STZ to induce DM.

The two groups were equally sub-divided into 2 sub-groups, according to the date of specimen collection.

Sub-group B1: 10 rats were sacrificed two weeks after establishment of DM.

Sub-group B2: 10 rats were sacrificed four weeks after establishment of DM.

\section{Induction of Diabetes}

1- Rats were left over-night fasting before receiving an IP injection of STZ (sigma chemical co., St. Louis, MO, USA). [50mg/kg body weight, diluted in $2 \mathrm{ml}$ of citrate buffer $\mathrm{pH} 4.6]$.

2- After 48 hours, blood sugar level was measured, animals were considered diabetic with serum glucose levels more than $233 \mathrm{mg} / \mathrm{dl}$ (Sajad et al, 2008).

At the end of each sub-group experimental period, the animals were weighed, and they were sacrificed by a high dose of anaesthesia. The upper and lower jaws were separated and the permanent molars of each rat were removed from bone, then they were split using bone chisel, to allow detachment of the pulp tissue intact. The left permanent molars pulps of each rat were processed, assigned for histological and immuno-histochemical examination. The pulps of the right permanent molars of each rat were assigned for ultra-structural examination.

\section{Examination Methods}

\section{Light Microscopic Examination:}

\section{a- For Routine Histological Examination:}

Half the number of the detached pulp tissue specimens were immersed in $10 \%$ formaldehyde solution for 48 hours. The specimens were dehydrated in graded series of ethanol, and immersed in xylene and then embedded in paraffin wax. Four micrometre thick sections were obtained and stained with $\mathrm{H} \& \mathrm{E}$.

\section{b. For Immunohistochemical Examination:}

\section{i. Specimen Preparation:}

1. The other half of pulp tissue specimens were fixed in $10 \%$ formaldehyde for only 8 hours, and then they were processed and imbedded in paraffin wax (care was taken so that the wax temperature did not exceed 60 degrees Celsius, in order not to affect the cell surface markers). 
2. For each tissue specimen, two paraffin sections, $4 \mu \mathrm{m}$ each were prepared, one for immunostaining with anti-CD3 and the other for immuno-staining with anti-CD20.

3. Sections were mounted on poly-L-lythene glass slides.

4. Sections were deparaffinised with xylene.

5. Sections were rehydrated in graded series of ethanol. Then, tissue sections were washed in a $10 \mathrm{mM}$ phosphate-buffered saline (PBS) bath for 10 minutes before starting the staining procedure.

\section{ii. Immuno-staining procedures:}

\section{1- Blocking endogenous peroxidase}

Slides were submerged in peroxidase quenching solution ( 1 part of $30 \%$ hydrogen peroxide added to 9 parts of absolute methanol) for 10 minutes.

The slides were washed with PBS for 2 minutes, 3 times.

\section{2- Exposure to Serum blocking solution:}

Few drops of serum blocking solution were added on each slide until they completely covered the tissue sections and they were incubated for 10 minutes.

Slides were not rinsed; instead, they were blot off solution.

\section{3- Exposure to the primary anti-body:}

Few drops of the primary anti-body were added on each slide until they completely covered the tissue sections.

Sections were incubated in moist chamber for 30-60 minutes, then rinsed with PBS for 2 minutes, 3 times.

\section{4- Exposure to biotinylated second antibody:}

Few drops of biotinylated second antibody were added on each slide until they completely covered the tissue sections and they were incubated for 10 minutes, and then rinsed with PBS for 2 minutes, 3 times.

\section{5- Exposure to the enzyme-conjugate:}

Few drops of ready-to-use streptavidin-peroxidase were added on each slide until they completely covered the tissue sections and they were incubated for 10 minutes.

Sections were rinsed with PBS for 2 minutes, 3 times.

\section{6- Exposure to the chromogen:}

AEC chromogen was prepared by mixing 3 drops of colour reagent buffer, 1 drop of enzyme substrate and 1 drop of AEC chromogen, in this order.

Several drops of the AEC chromogen were placed on each slide and incubated in a moist chamber at room temperature for 20 minutes.

Then, slides were immersed in 2 consecutive washes of PBS, each for 3 minutes.

\section{7- Counter staining:}

The slides were counter stained using haematoxylin.

Then, they were washed with tap water.

8- Mounting: Slides were mounted using aqueous mounting medium then cover slips were put in place.

\section{iii- Statistical Analysis}

The positive CD3 and CD20 cells were counted under a power (x 200) lens, for both the control and diabetic groups. The obtained data were tabulated and ANOVA test was used to compare between the different groups concerning the number of positive cells. 


\section{Ultra-structural Examination:}

The pulp tissues were sliced into $1 \mathrm{~mm}$ cubes using sharp lancet on dental pink wax. Specimens were primarily fixed in buffered formaldehydegluteraldehyde solution with pH7.4 (karnovsky's solution) overnight in temperature $4 \mathrm{C}$, post-fixed in buffered $1 \%$ osmium tetroxide solution $\left(\mathrm{OSO}_{4}\right)$ for not more than 1.5 hours.

The tissue samples were dehydrated in graded series of ethanol, and embedded in low viscosity resin (Spurr). Semi-thin sections were obtained, stained with toluidine blue and examined under light microscope to choose areas of interest.

Ultra-thin sections were cut, stained with urenyl acetate and lead citrate and then were examined by Transmission Electron Microscope (TEM) in the Electron Microscopy Unit of the Veterinary Hospital of Armed Forces.

\section{RESULTS}

\section{Histological Results}

\section{Group A (Control Group):}

Examination of the H\&E-stained sections of both sub-groups A1 and A2, showed normal architecture of the pulp with regularly aligned odontoblastic zone, obvious cell-free zone (in coronal pulp) followed by cell-rich zone, where the cell density was apparently high. Endothelially lined blood vessels were seen in the pulp core, filled with RBC's (Fig.1a). Pulp core showed normal appearance of loose fibrillar connective tissue elements, spindleshaped fibroblasts with basophilic cytoplasm \& intermingled with collagen fiber bundles (Fig.1b).

\section{Group B (Experimental Group)}

\section{Sub-group B1 (2-Week diabetes):}

Histological examination of H\&E-stained sections of pulp tissue of this group showed irregular odontoblastic zone in which some areas showed total loss of odontoblasts. The cell-free zone was hardly observed and the cell-rich zone could not be identified from pulp core. The pulp core showed, irregularly arranged fibroblasts and less cellular density compared to the control group. The blood vessels were hardly seen, and they showed marked narrowing of their lumen with irregular endothelial lining (Fig.1 c,d ).

\section{Sub-group B2 (4-week diabetes):}

Examination of H\&E-stained sections of the pulp tissue of this group showed irregularly arranged flattened odontoblasts, with absence of cells in some areas. The cell free zone as well as the cell rich zone was totally ill defined. Cytoplasmic vacuolations were obviously seen in the pulp. Fibroblasts and collagen fibres were irregularly arranged. Some areas in pulp core showed hyalinization, the blood vessels were collapsed and extravasated RBCs were clearly detected in some areas (Fig 1 e,f)

\section{Ultra-structural Results}

\section{Group A (Control Group):}

Ultrastructural examination of pulp sections of both sub-groups A1 (2 weeks) and A2 (4 weeks) showed adjacent odontoblasts with open-faced nuclei, regular, double-layered nuclear membranes, homogeneous marginated chromatin, and abundant regular RER in the perinuclear area (Fig. 2a). The cytoplasm showed many mitochondria with normal appearance of internal cristae. In the cellrich zone, fibroblasts appeared stellate shaped with oval nuclei and regular nuclear membranes. (Fig 2b). They had regular cytoplasmic organelles in a moderately electron-dense cytoplasm many RER cisternae as well as mitochondria. Well-developed collagen fibers appeared with distinct cross-banding (Fig. 2c). The pulp core showed blood vessels with patent lumina lined by endothelial cells that appeared 
with normal nuclear membranes and homogeneous chromatin. Their basal laminae showed regular thickness (Fig. 2d). Lymphocytes were seen in the pulp core having rounded nuclei with regular nuclear membranes. The nucleus almost filled the cell body leaving only thin rim of cytoplasm around it (Fig. 2e). Many myelinated nerves with intact myelin sheath and regular structure were observed in pulp core. On higher magnification, regular lamination of myelin sheath was observed. Some nerves showed well developed Schwann cells embracing the nerves (Fig. 2f).

\section{Group B (Experimental Group):}

\section{Subgroup B1 (2-week diabetes):}

Ultra-structural examination of pulp sections of this group revealed that the odontoblasts lost orientation with the presence of wide extracellular compartments and few cytoplasmic vacuolations. Some odontoblasts showed irregular nuclear configurations with peripheral chromatin condensation. Many RER appeared dilated and mitochondria were degenerating possessing granular internal structure (Fig. 3a). Fibroblasts showed irregular nuclei with electron-dense chromatin, as

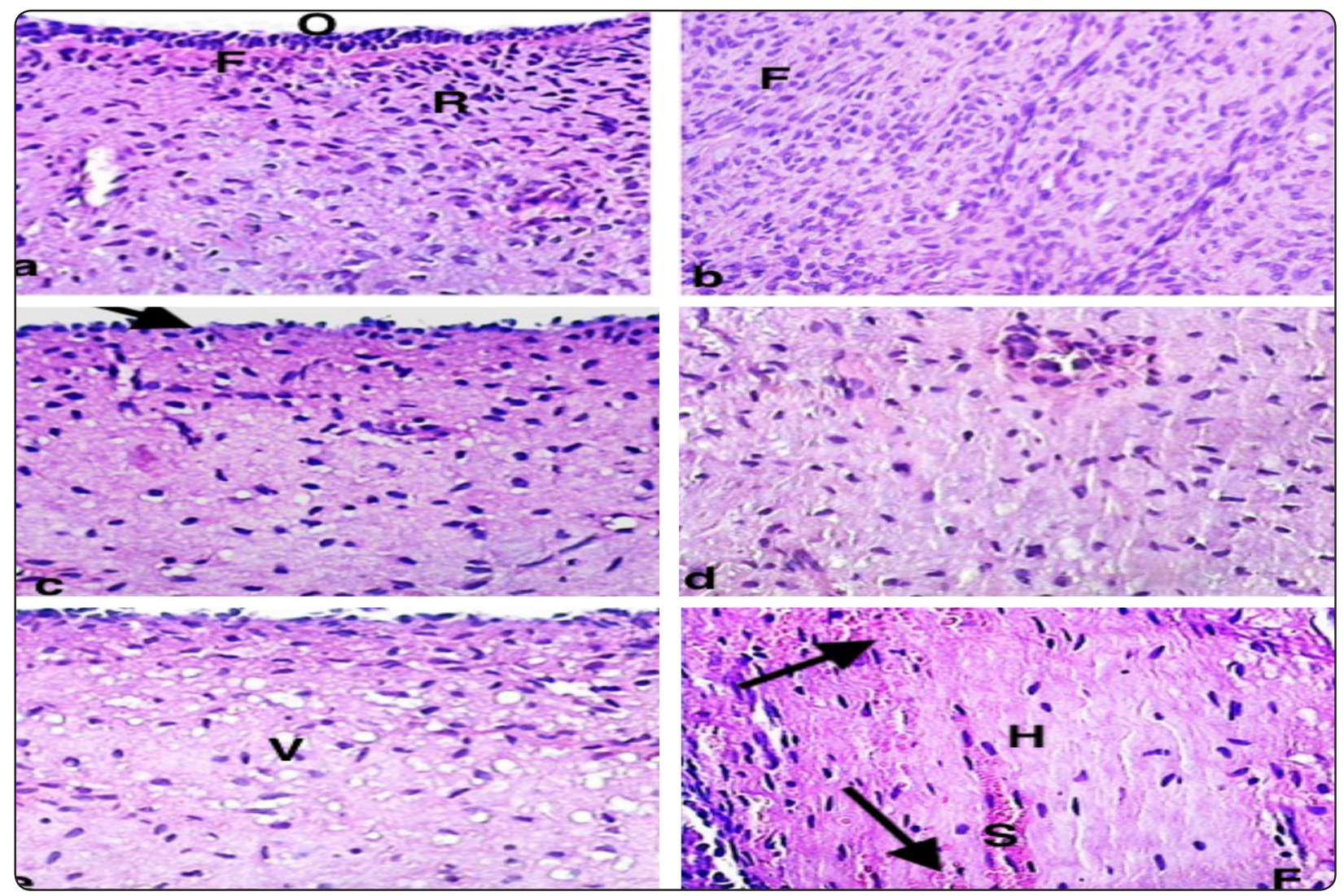

Fig. (1): A photomicrograph of pulp core of (Group I), (H\&E x 200). a) Control group (Group A) showing: pallisaded pattern of odontoblasts at the pulp periphery $(\mathrm{o})$, cell-free zone $(\mathrm{F})$, and the cell-rich zone $(\mathrm{R}) \mathrm{b})$ showing regularly arranged fibroblasts $(\mathrm{F})$ that intermingled with collagen fibre bundles $(\mathrm{C} \mathrm{c})$ subgroup B1: showing irregular odontoblastic zone, absence of cell-free zone and cell-rich zone. d): subgroup B1: showing markedly less cellular density in the pulp core, and a blood vessel with narrowed lumen (arrow). e) subgroup B2, showing irregularly arranged flattened odontoblasts with loss of cells in some areas. Ill-defined cell-free zone as well as cell-rich zone, Cytoplasmic vacuolizations (V) f) subgroup B2: showing hyalinized areas $(\mathrm{H})$, collapsed blood vessels $(\mathrm{S})$ with exravasation of RBCs (arrows) irregularly arranged fibroblasts and collagen fibers. (H\&E x 200 ). 


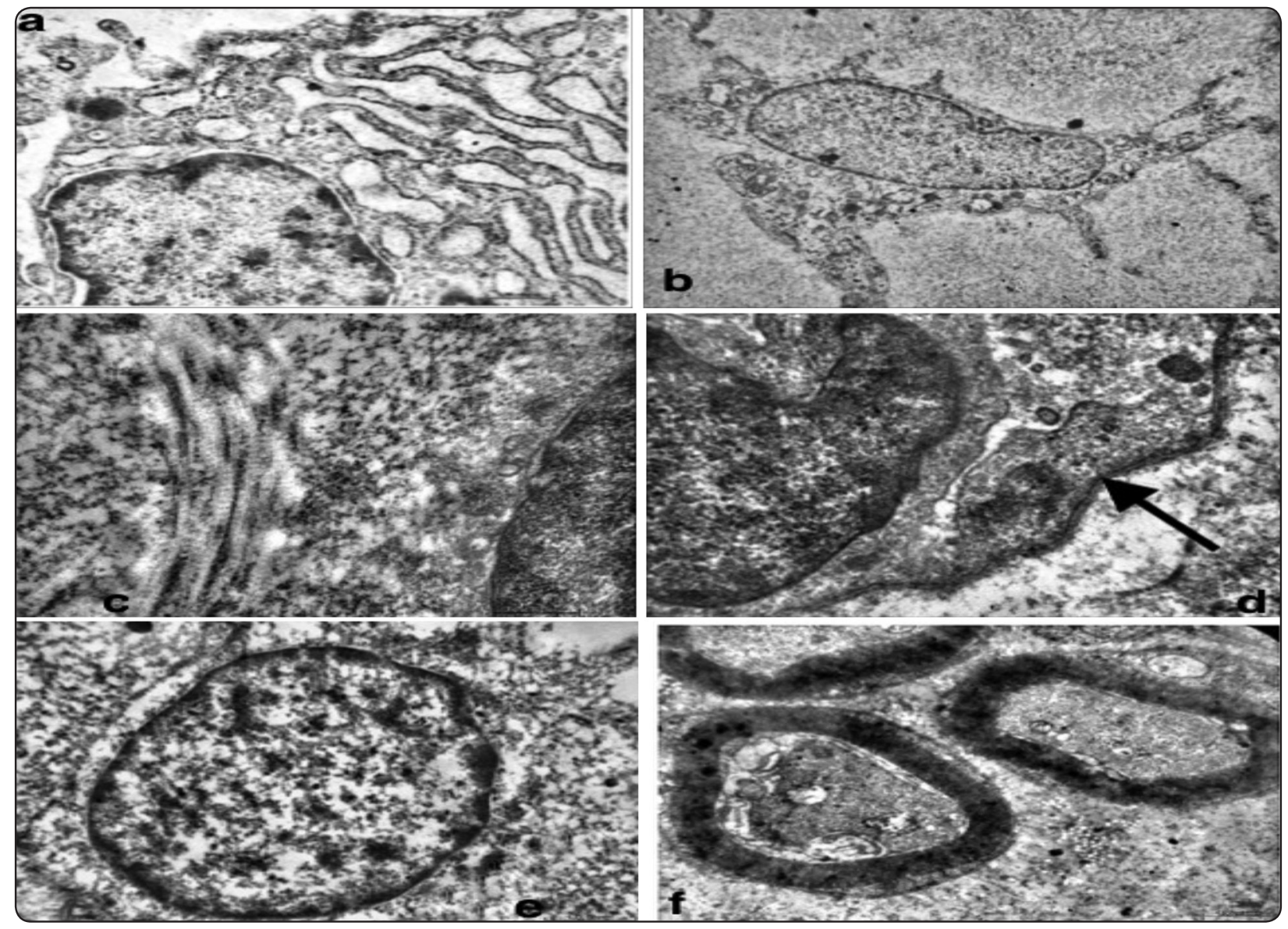

Fig (2): Electron micrograph of Group (A) showing: a) an odontoblast with open-faced nucleus with peripheral chromatin condensation and regular nuclear membrane, abundant RER perinuclearly. (x 15000). B) Stellate shaped fibroblast with regular nuclear membrane and chromatin distribution (x 8000). C) Collagen fibers with distinct cross banding, adjacent to a fibroblast. (x 20000) d) Basal lamina around the endothelial cells (arrow). (x15000). e) lymphocyte with rounded nucleus almost filling the cell. (x 10000). f) nerves with intact myelin sheaths. (x8000).

well as widening and irregularity of the perinuclear space. Cytoplasmic vacuolations were observed in some cells. The mitochondria of the fibroblasts had lost their regular internal cristae, the RER appeared almost normal in most of the cells, few were dilated (Fig. 3b).

In the pulp core, few apoptotic fibroblasts, were detected. Collagen fibers appeared with low electron density and loss of regular cross banding (Fig. 3c). The blood vessels appeared to be obliterated or collapsed and their basal laminae were markedly thickened. Nuclei of the endothelial cells, lining some blood vessels showed irregular nuclear membrane (Fig. 3d). Lymphocytes were found near the blood vessels, and they had rounded nuclei occupying major part of the cell body, with ill-defined cell membrane (Fig. 3e). Myelinated nerves were surrounded with thin irregular myelin sheaths that were disrupted in some areas (Fig. 3f).

\section{Sub-group B2 (4-week diabetes):}

Ultra-structural examination of pulp sections of this group showed widely separated, shrunken odontoblasts Some showed definite signs of pyknosis, where their nuclei appeared electron-dense with 


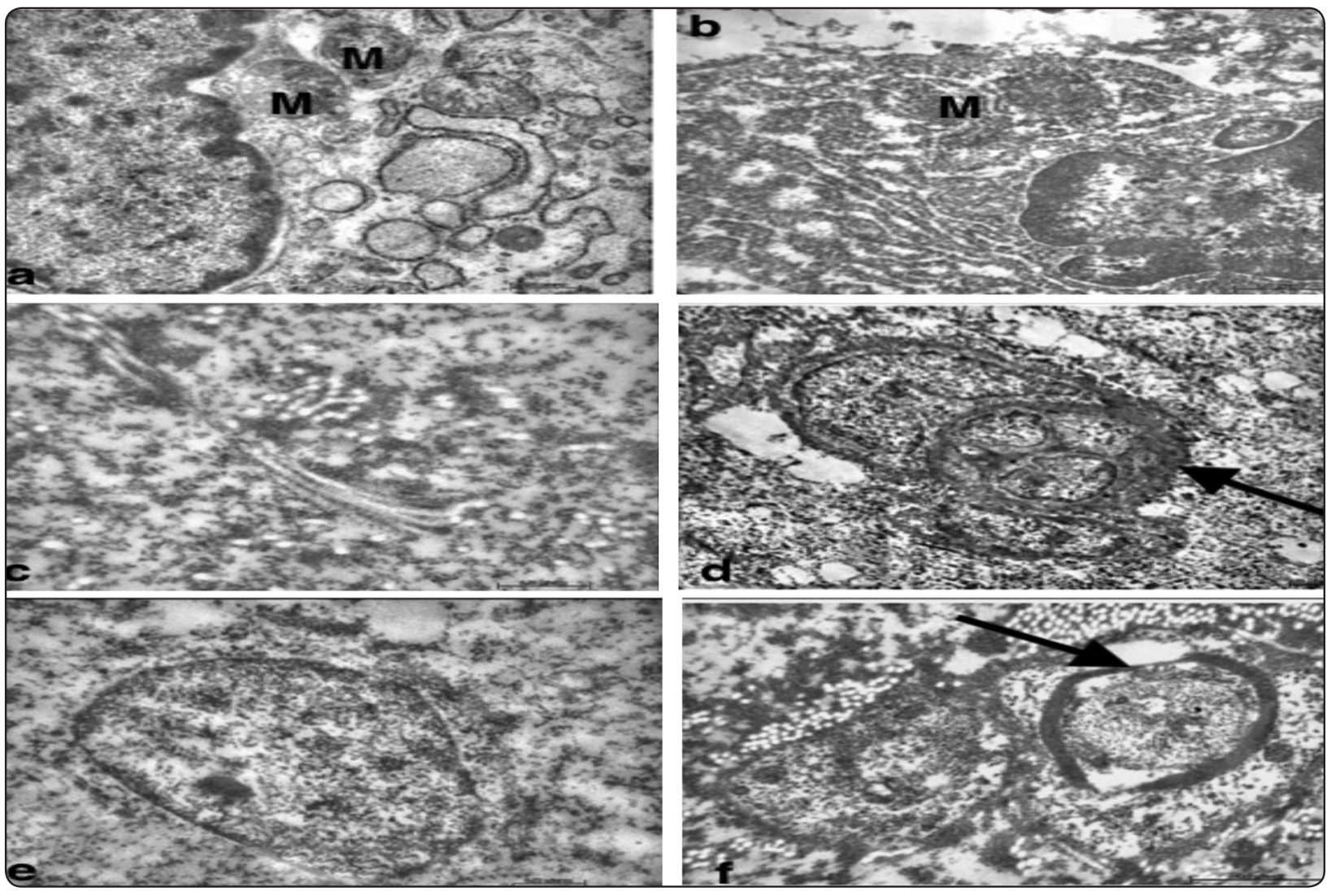

Fig (3): Electron micrograph of subgroup B1 showing: a) odontoblast with dilated RER and mitochondria with granular internal structure(M). (x 15000). b) fibroblast with irregular nucleus and mitochondria (M) with lost internal cristae. (x10000). c) electrolucent collagen fibres with indistinct cross banding. (x 20 000). d) an obliterated blood vessel with markedly thickened basal lamina (arrow). A pericyte (P) embracing the vessel. (x 4000). e) lymphocyte with ill-defined cell membrane (x 15000). f) thinning and loss of continuity of the myelin sheath (arrow). (x 15000).

irregular membranes. The nuclei of some odontoblasts showed corrugated nuclear borders with margination and clumping of chromatin, cytoplasmic vacuolations were more apparently increased in size and in number in most cells. Areas of dilated RER with loss of ribosomes were observed. Mitochondria had lost their internal cristae and some were completely degenerated (Fig. 4a). In the pulp core, the fibroblasts showed numerous cytoplasmic vacuolations with electron dense nucleus and cytoplasm and dilated RER. Apoptotic fibroblasts with irregular, shrunken, and electron dense nuclei, were detected in some specimens (Fig. 4b). Few single collagen fibers with indistinct cross banding were detected (Fig. 4c). Blood vessels showed massive destruc- tion, in which there were numerous vacuolations in the cytoplasm of endothelial cells, with nearly occluded lumen. Also, many extracellular RBCs were detected, denoting destruction of blood vessels (Fig. 4d). Lymphocytes appeared with karyolitic nuclei (Fig. 4e). Many nerves showed partial loss of their myelin sheaths (Fig.4f)

\section{Immuno-histochemical Results}

\section{Group A (Control Group):}

The examination of pulp sections of the control groups which were immunostained with anti-CD3 showed that T-lymphocyte cells were positively stained, appearing with brownish cytoplasm. 

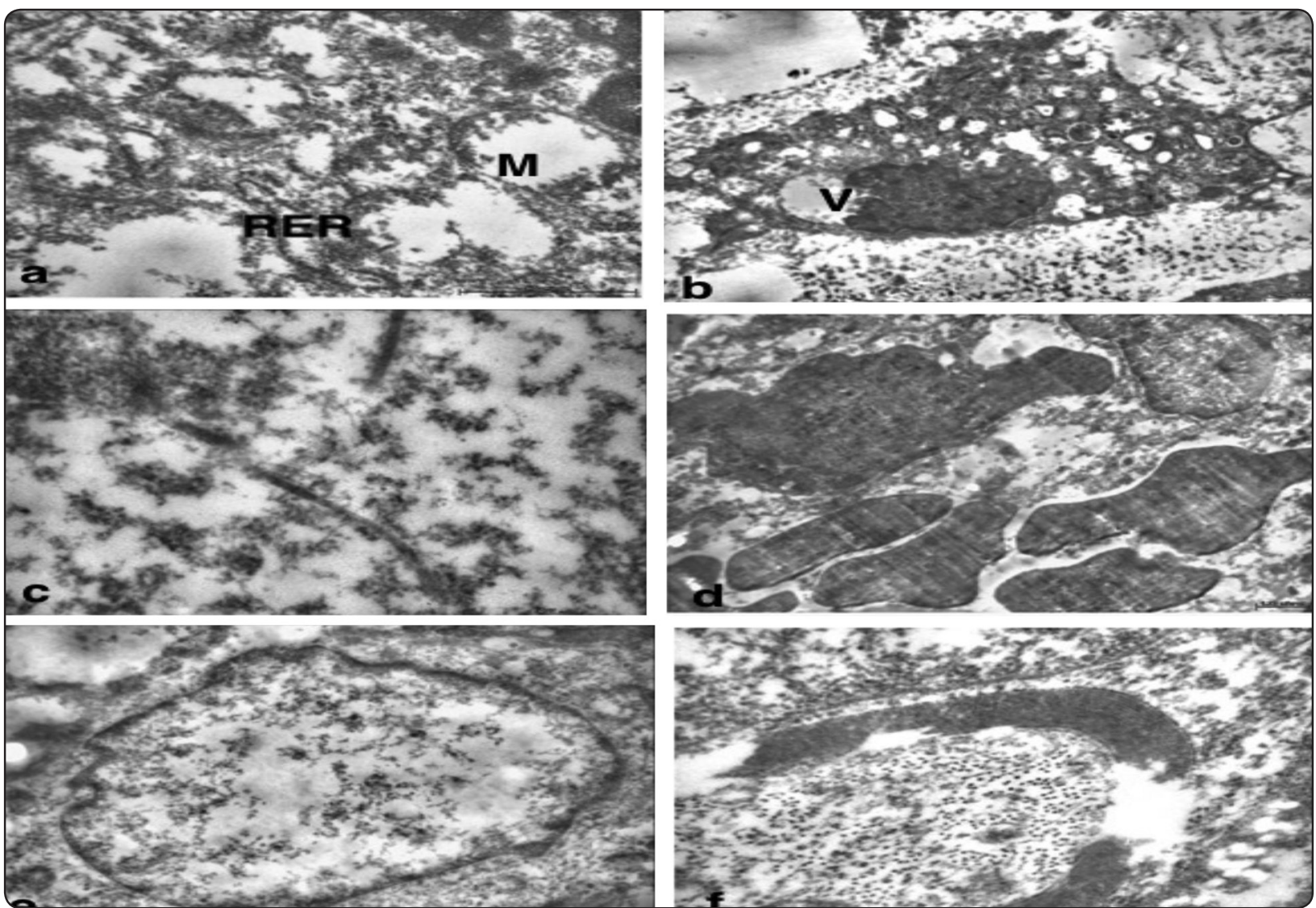

Fig(4): Electron micrograph of subgroup B2 showing: a) odontoblasts with dilated RER, degenerated mitochondria (M) and granular cytoplasm. (x 20000 ). b) a fibroblast with numerous cytoplasmic vacuolations with a large one(V) compressing the electron dense corrugated nucleus. (x 6000).c) sparse collagen fibres with indistinct cross banding. (x 30000).d) extravasated RBCs. (x 6000) .e) lymphocyte with karyolitic nucleus and corrugated nuclear membrane. (x10000).f) partial loss of myelin sheath. (x 30000).

They were abundant in the pulp core region especially near the blood vessels. The rest of pulp cells showed negative reaction and were stained blue (Fig. 5a). Also, examination of pulp sections immunostained with anti-CD20, revealed positively stained B-lymphocytes, with brown cytoplasmic reaction while the rest of pulp cells were negatively stained. The B-lymphocytes were less abundant than T-lymphocytes and were also found in the pulp core region, near the blood vessels (Fig. 5b).

\section{Group B (Experimental Group):}

\section{Subgroup B1 (2 weeks DM):}

Examination of immunostained sections for both $\mathrm{CD} 3$ and $\mathrm{CD} 20$ cells of this group revealed few positively stained T-lymphocytes (Fig. 5c) and B-lymphocytes (Fig. 5d) respectively. The T\&B lymphocytes were found near the blood vessels in the pulp core region.

\section{Subgroup B2 (4 weeks DM):}

Examination of immunostained sections for CD3 and CD20 cells revealed the rare presence of positively stained T-lymphocytes (Fig. 5e) and B-lymphocytes (Fig. 5f) respectively in the pulp core near the blood vessels. 


\section{Statistical Results}

Using one-way ANOVA, there was a highly significant difference between groups in CD 20 positive cells $(\mathrm{f}=37.3, \mathrm{p}<0.001)$. Post-Hoc LSD test showed a highly significant decrease in CD 20 positive cells in both DM 2 weeks and DM 4weeks as compared to control $(\mathrm{p}<0.001)$. There was no significant difference between DM 2weeks and DM 4 weeks ( $p>0.05)$. Also, using one-way ANOVA, there was a highly significant difference between groups in CD3 positive cells ( $\mathrm{f}=21.1, \mathrm{p}<0.001)$. Post-Hoc LSD test showed a highly significant decrease in CD 3 positive in both DM 2weeks and DM 4weeks as compared to control $(\mathrm{p}<0.001)$. There was no significant difference between DM 2 weeks and DM 4weeks ( $>00.05)$. The mean \& standard deviation of the number of positive cells of all groups were presented in table I, and the mean of positive cells was also presented in the following histogram.

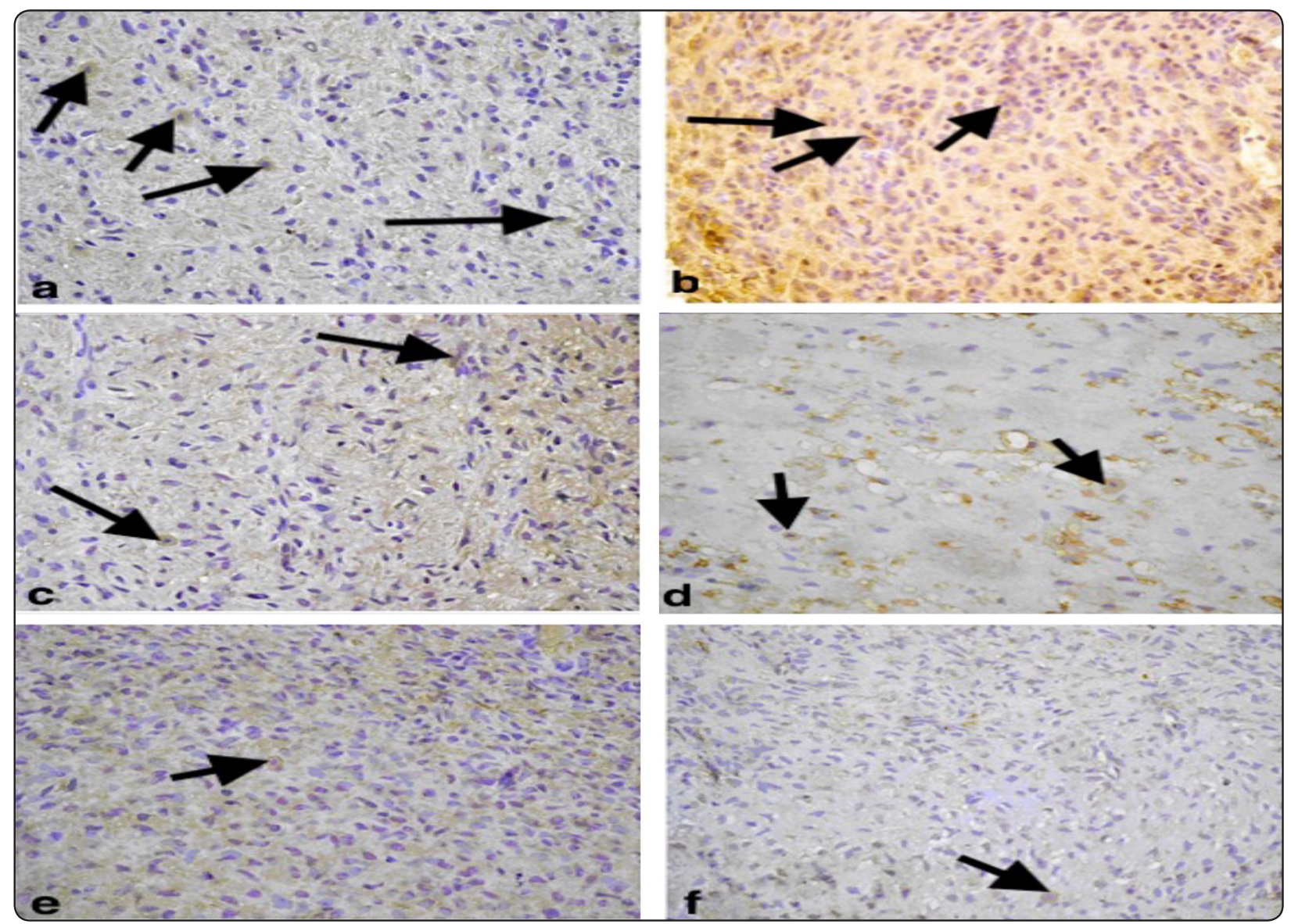

Fig. (5): A photomicrograph of the pulp core of a) Group A showing many CD3 positive cells (arrows). (x 200) b) Group A with many CD20 positive cells in the pulp core.(arrows). (x 200) c) subgroup B1 with rare positively stained CD3 positive cells in the pulp core (arrows). (x 200). d) subgroup B1 showing rarely detected CD20 positive cells in the pulp core (arrows).(x 200). e) subgroup B2 showing rarely detected CD3 positive cells in the pulp core (arrow). (x 200). f) subgroup B2, showing rarely detected CD20 positive cells in the pulp core (arrow). (x 200). 
TABLE (I) Showing the mean \& standard deviation of $\mathrm{CD} 20$ and $\mathrm{CD} 3$ positive cells in the different groups

\begin{tabular}{|c|c|c|c|}
\hline & Control & Diabetes 2wk & Diabetes 4wk \\
\hline CD 20 & $8.83 \pm 2.56$ & $2.28 \pm 1.25$ & $1.22 \pm 1.39$ \\
\hline CD 3 & $11.62 \pm 5.20$ & $2.50 \pm 0.54$ & $2.25 \pm 0.88$ \\
\hline
\end{tabular}

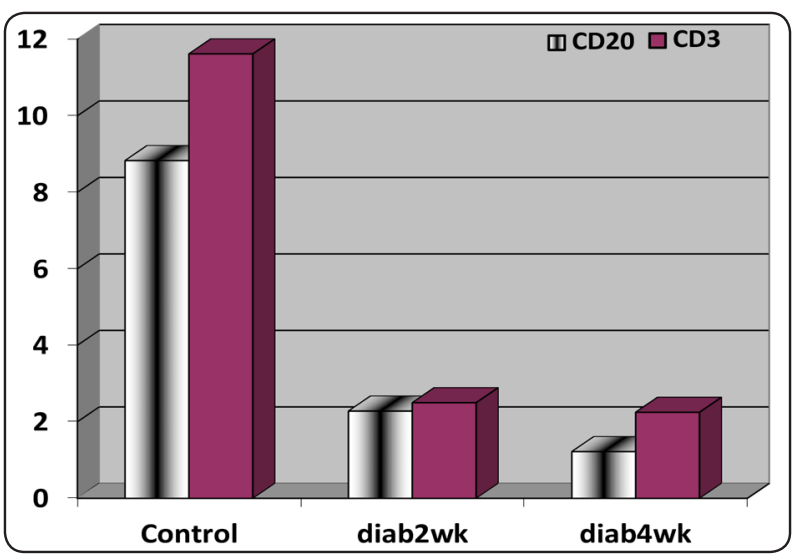

Fig. (6): Histogram showing the mean of CD20 and CD3 positive cells in the different groups.

\section{DISCUSSION}

Diabetes mellitus is a metabolic disorder characterized by relative or absolute insufficiency of insulin along with disturbances of carbohydrate, fat and protein metabolism. Many oral diseases have been associated with hyperglycaemia such as periodontitis, dental caries etc. Patients with diabetes mellitus have a rapidly ageing pulp due to obliterative endartities (Pratha and Jayalakshmi, 2016). The dental pulp was the tissue of choice for our study because of its nature as a delicate connective tissue with limited or no collateral circulation (Shabahang, 2013). Albino rats were chosen as the experimental animals because of their convenient handling, they were readily available, and they possessed sufficient amount of pulp tissue within their teeth, especially molars. Also, STZ was chosen to induce DM because it is well known for its selective pancreatic islet-Beta-cell cytotoxicity. In addition it was better tolerated by experimental animals and had lower mortality rates than Alloxan (Tszkudelski, 2001; Lenzen, 2008) .

The histological results of the present study revealed some changes in the pulp elements of subgroup B1 (2-weeks diabetic rabbits), such as irregular odontoblastic zone with loss of palisading pattern, and focal loss of odontoblast cells. The cellrich-zone showed apparently less cellular density than in control group. Those findings might be due to hyperglycaemia-associated reduction in oxygen tension of the tissues, leading to reduction in the proliferating capacity of the cells, and increased cell apoptosis, matching those results of (Sajed et al., 2008). Also, the AGEs accumulation, which accompany DM, enhances the oxidant stress with subsequent enhancement of apoptotic changes or reduced cellular proliferation (Southerland et al., 2005; Leite et al., 2008).

The results of this study also revealed the presence of wide intercellular compartments with hyalinization in Subgroup B2 (4 weeks) indicating defective collagen content, this matched with the results of several authors who reported that DM significantly depresses skin collagen hydroxyproline content resulting in a marked reduction in collagen synthesis. The defective collagen content in our study could be also attributed to the enhanced stimulation of MMPs resulting from over production of ROS that accompany DM (Lijima, 2007; Leite et al., 2008). The MMPs are zinc-dependent endopeptidases, that are capable of cleaving most of basement membranes and extracellular matrix components, including collagen, fibrin, laminin, and proteoglycans, in the connective tissues (Nelson et al., 2000).

Also the same group showed exaggerated features of distorted pulp architecture, and marked apoptotic changes of all pulp cells. These 
exaggerated effects can be attributed to the longstanding state of oxidative stress, which lead to apoptosis of cells, and activation of MMPS which destroys the extracellular matrix elements especially collagen.

Blood vessels of the same group were rarely found, and in most of the cases they appeared occluded, matching those findings of Southerland, et al, (2005) who reported that the AGEs accumulation, accompanying long standing DM, have been associated with enhanced oxidant stress and subsequent apoptosis of endothelial cells, thickening and altered function of the basement membranes of blood vessels and capillaries.

The pulp core of the same group showed wider intercellular spaces as well as different-sized vacuolations in the cytoplasm of odontoblasts and fibroblasts. This can be interpreted as degenerative changes and evidence of apoptosis. These degenerative changes could be related to alteration in the lipid metabolism in various tissues due to DM, resulting in over production of ROS and creating a state of oxidative stress in various cells of the body. Most of pulp vessels in our study were collapsed and extravasation of RBCs was clearly detected in many areas, indicating weakened structure of the blood vessels wall. This came in agreement with Bender et al., (2003), who reported that the blood vessels of diabetic rats were damaged due to accumulation of atheromatous deposits in the intimal layer of the blood vessels lumen. This alters the vessels tone and affects its patency.

Ultra-structural results of this study confirmed the histological findings and revealed wide range of degenerative changes affecting almost all pulp elements. In sub-group B 1, the nuclei of odontoblasts and fibroblasts showed irregular nuclear membranes, widened irregular perinuclear space, areas of electron-dense chromatin aggregations, as well as margination of chromatin. These were considered as initial signs of pyknosis or apoptosis as reported by Robinstein et al., (2008); Leite et al., (2008).
It was also reported by Leite et al., (2008), that DM significantly lowers the tissue levels of sialic acid, which is a potent anti-oxidant naturally occurring in most of body tissues, by acting as hydrogen ions scavenger, that is oxidized by hydrogen peroxide and converted into a stable nontoxic compound, so reduction of sialic acid by DM results in enhanced oxidative stress in various body tissues.

The cytoplasm of odontoblasts and fibroblasts showed numerous different-sized vacuolations which may be a sign of degeneration. These vacuoles might be autophagic vacuoles that contained dead cellular organelles before hydrolizing them. Mitochondria appeared with granular internal structure and in most cases showed loss of its cristae. This finding can be explained by the fact of the cytotoxic effect of the ROS to the mitochondria, which attacks the mitochondrial DNA, thus impairing mitochondrial metabolism (Tszkudelski 2001; Lenzen, 2008).

In our study, the RER in odontoblasts and fibroblasts, in most specimens were dilated indicating a degenerative process that might further explain this markedly low level of collagen fibres observed in this study.

Collagen fibers of the diabetic groups of this study, appeared sparse, or even as single fibers. This could result from the retarded collagen synthesis combined with the enhanced collagen degradation by the stimulation of MMPs. This matched what was reported by Bender et al., (2003); Manfredi et al., (2004) ; Southerland et al.,(2005); Silva et al., (2007); Leite et al., (2008).

In our study, collagen fibers also appeared electroluscent with indistinct cross banding and this was explained by Lien et, al, (1984) who reported that glucose inhibits collagen fibril formation in vitro. Nonfibrillar collagen cannot be cross-linked by lysyl oxidase, an enzyme that catalyzes the initial cross-linking reaction. Collagen that is not cross-linked is unstable and more susceptible to collagenolytic attack. 
Obliterated and collapsed blood vessels with markedly thickened basal laminae were observed in both diabetic sub-groups of this study. The nuclei of endothelial cells showed apoptotic changes as irregular nuclear membranes and hyper chromatic nuclei. This is in agreement with what was reported by Vlassara et al., (2002); Bender and Bender, (2003); Manfredi et al., (2004); Southerland et al., (2005).

In the current study, lymphocytes of the diabetic groups were rarely detected, found mainly close to the blood vessels. Their cell membranes were illdefined and their nuclei were karyolitic, indicating signs of their degeneration. This finding was proved and explained by Manfredi et al, (2004) and Robinstein et al., (2008) who reported that hyperglycaemia and its consequent oxidative stress diminished cell viability and induced apoptosis of immune cells. A number of reviews and studies have proposed mechanisms to explain the relationship between DM and periodontal disease, including: microvascular disease, changes in components of gingival crevicular fluid, changes in collagen metabolism, an altered host response, altered subgingival flora, genetic predisposition. In addition, in vitro studies of monocytes from people with DM have shown a hyper responsive phenotype with over expression of pro-inflammatory mediators such as IL-1 $\beta$, IL1-6, platelet-derived growth factor, $\mathrm{TNF} \alpha$, and prostaglandin E2. In similar in-vivo studies, patients with periodontitis and diabetes were found to have significantly higher levels of local inflammatory mediators compared to systemically healthy individuals with periodontal disease (Southerland et al., 2005; Silva et al., 2007). Evidence has accumulated supporting a role for AGEs in exacerbating diabetic systemic complications and periodontal disease severity associated with a chronic and intense inflammatory response. Moreover, AGEs have been associated with enhanced oxidant stress, and subsequent expression of endothelial cell adhesion molecule
(Southerland et al., 2005; Leite et al., 2008). All of this explains the role of DM in the development of immune deficiency.

Interestingly, the present study showed affection of most myelinated nerves in the form of thinning andlor rupture of their myelin sheaths in both diabetic sub-groups. This could be attributed to the hypoxia and increased dependence on anaerobic metabolism, resulting in nerve fibre degeneration. This finding also came in agreement with what was reported by Sajad et al., (2008) and Kellogg et al., (2009) who found that DM caused oedema and degenerative changes in neurons and purkinji cells in cerebellum.

Ultra-structural results of sub-group B2 showed exaggeration of the previous findings, indicating that the DM-associated damaging effects on pulpal structures were time dependent, as agreed upon by Collin et al., (2000); Atkinson et al., (2001); Schmitt et al., (2006); Sajad et al., (2008).

The immuno-histochemical results of this study revealed a marked reduction of $\mathrm{T}$ and $\mathrm{B}$ lymphocytes in sub-group B1 as detected by the few positively stained cells for CD3 and CD20 respectively, when compared to the control group. Further reduction of T\&B lymphocytes in subgroup B2 was noticed so that these cells were rarely detected. The statistical analysis revealed significant decrease in T\&B lymphocytes in group B1 \& B2. These findings came in agreement with what was reported by Robinstein, et al., (2008) about the effect of hyperglycaemia on the viability and proliferation of $\mathrm{T}$ and $\mathrm{B}$ lymphocytes in the lymph nodes and spleen of diabetic mice. They reported a significant time-and-dose-dependent decrease in $\mathrm{T}$ and $\mathrm{B}$ cell proliferation and viability. Also, they reported that DM increased apoptosis and induced oxidative stress which is specifically toxic to the lymphocytes and lead to damage of their cellular components, and that might account for the marked immune deficiency that accompanies DM. 


\section{CONCLUSIONS}

1. DM led to degenerative changes of the pulp elements namely, cells, fibers, nerves, and vessels.

2. DM also led to affection of T\&B lymphocytes of the pulp.

3. DM-associated damaging effects on various pulpal structures were time-dependent, which means that long standing hyperglycaemia aggravated the tissue destruction.

4. The pulpal changes accompanying DM can account for the decreased sensitivity, retarded healing capacity of the pulp, and increased susceptibility to infection that may lead to pulp death.

\section{REFERENCES}

- Atkinson M.A. and Eisenbarth G.S.: Type I Diabetes, New perspectives on disease pathogenesis and treatment . Lancet 358:221-229, 2001.

- Bender I. B. and Bender A. B : Diabetes Mellitus and the Dental Pulp. J Endod, 29: 383-492, 2003.

- Bingly P. J., Bonifacio E., and Ziegler A. G. : Proposed guidelines on screening for risk of type I diabetes . Diabetes Care, 24: 398, 2001.

- Catanzaro O., Dziubecki D., Lauria L . Ceron C. M., and Rodriguez R.: Diabetes and its effects on dental pulp. Journal of Oral Sience , 48: 195-199, 2006.

- Collin H. L., Niskanen L., and Uusitupa M. et al. :Oral symptoms \& signs in elderly patients with type 2 diabetes mellitus. Oral Surg Oral Med Oral Pathol Oral Radiol Endod. 90: 299-305, 2000.

- Iwama A., Taisuke M. , Masahito T. , Koki N. , Naoya H. , Ichiro I. , Naoki S. , Masahiro Y. , and Hiroshi N.: Increased number of anaerobic bacteria in the infected root canal in type 2 diabetic rats. Oral Surg Oral Med Oral Pathol Oral Radiol Endod. 101: 681-687, 2006.

- Kellogg A.P., Converso K. , Wiggin T., Stevens M., and Pop-Busui R. : Effect of cyclooxygenase-2 gene inactivation on cardiac autonomic left ventricular function in experimental diabetes. Am J Physiol Heart Circ Physiol 296 : H453-H461, 2009.

- Leite M. F., Ganserla E., Marques M., and Nicolau J.:
Diabetes induces metabolic alteration in dental pulp. J Endod , 34:1211-1214, 2008.

- Lenzen S. : The mechanism of alloxan and streptozotocininduced diabetes. Diabetologia, 51(2):216-26, 2008.

- Lijima R. : Characterization of the reaction between sialic acid (N-acetylnuramic acid) and hydrogen peroxide. Biol Pharm Bull, 30:580-582, 2007.

- Manfredi M., McCullough M. J., , Vescovi P. ,Al-Kaarawi Z. M., Porter S. R.: Updates on diabetes Mellitus and related oral diseases. Oral Diseases, 10:187-200, 2004.

- Nelson A. R., Fingleton B., Rothenberg M. L., and Matrisian L. M.: Matrix Metalloproteinases: Biologic Activity and Clinical Implications. J Clin Oncol 18:1135-1149, 2000.

- Pratha A, Jayalakshmi A. S. Relationship between Diabetes Mellitus and Pulpal Infection \& Periapical Diseases et al /J. Pharm. Sci. \& Res. Vol. 8(8), 794-796. 2016,

- Robinstein R., Genaro A. M. , Motta A., Cremaschi G. and Wald M. R. : Impaired immune response in Streptozotocininduced type I diabetes in mice. Involvement of high glucose. British Society for Immunology, Clinical and Experimental Immunology, 154:235-246, 2008

- Sajad H.M., Abdul Baqui M., Bahgat R.C., Darzi M.M., and Abdul wahid S.: Biochemichal and Histomorphological study of Streptozotocin-induced Diabetes in Rabbits. Pakistan Journal of Nutrition, 7(2):359-364, 2008.

- Schmitt A. , Bigl K. , Meiners I., and Schmitt J. : induction of reactive oxygen species and cell survival in the presence of advanced glycation end products and similar structures. Biochem Biophys Acta, 1763: 927-36, 2006.

- $\quad$-Shahrokh Shabahang Treatment options: apexogenesis and apexification. Pediatr Dent .35(2):125-8. 2013

- Silva J. A. F. , Lorencini M. , Peroni L. A., De La Hoz C. L. R., Carvalho H. F. , and Stach-Machado D. R. : The influence of type I diabetes mellitus on the expression and activity of gelatinases (matrix metalloproteinases-2 and -9) in induced periodontal disease. Journal of periodontal research, 43: 48-54, 2007.

- Southerland J. H., Taylor G. W., and Offenbacher S.: Diabetes and Periodontal Infection: Making the Connection. Clinical Diabetes, 23: 171-178, 2005.

- Tszkudelski T.: The Mechanism of Alloxan and Streptozotocin Action in B Cells of the Rat Pancreas. Physiol. Res. 50:536-546, 2001

- Vlassara H., and Palace M. : Diabetes and advanced glycation end products. J Intern Med 251:87-101, 2002. 